\title{
TTR
}

Traduction, terminologie, re?daction

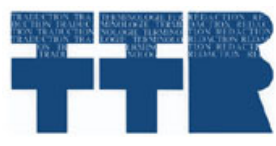

\section{Émile Zola devant la censure victorienne}

\section{Denise Merkle}

Volume 7, numéro 1, 1er semestre 1994

Genres littéraires et traduction

URI : https://id.erudit.org/iderudit/037169ar

DOI : https://doi.org/10.7202/037169ar

Aller au sommaire du numéro

\section{Éditeur(s)}

Association canadienne de traductologie

\section{ISSN}

0835-8443 (imprimé)

1708-2188 (numérique)

Découvrir la revue

\section{Citer cet article}

Merkle, D. (1994). Émile Zola devant la censure victorienne. TTR, 7(1), 77-91. https://doi.org/10.7202/037169ar

\section{Résumé de l'article}

Emile Zola devant la censure victorienne - Cette étude donne un aperçu sommaire de quatre formes prises par la censure victorienne - la ligue de moralité ( National Vigilance Association »), les bibliothèques de prêt ( " circulating libraries »), la justice et l'autocensure (" bowdlerism ») — et leurs effets sur la traduction des romans de Zola. Les oeuvres de ce dernier ont connu un succès de scandale et ont provoqué un débat journalistique en France, mais Zola n'a jamais été traîné en justice à cause de ses écrits. Par contre, en Angleterre, les cercles littéraires établis honnissent les traductions victoriennes de ses oeuvres et la ligne de moralité appuyée par le moraliste Stead lance une campagne de censure des traductions de Zola. L'éditeur de ces traductions, Henry Vizetelly, a dû comparaître deux fois devant les tribunaux et les deux fois il a été condamné. Non seulement il dut payer une amende et passer trois mois en prison, mais encore Nana, Piping Hot! (Pot-Bouille) et The Soil (la Terre) ont été interdits, même si ces trois traductions avaient - comme les autres - été « autocensurées » par les traducteurs et l'éditeur avant la parution. Il est dès lors évident que ce sont les idées réformatrices de Zola et le lectorat visé (petite bourgeoisie et classes populaires) qui ont motivé l'opposition des pouvoirs constitués de l'Angleterre de l'époque aux romans de Zola.
Tous droits réservés (C) TTR: traduction, terminologie, rédaction — Les auteurs, Ce document est protégé par la loi sur le droit d’auteur. L’utilisation des 1994 services d'Érudit (y compris la reproduction) est assujettie à sa politique d'utilisation que vous pouvez consulter en ligne.

https://apropos.erudit.org/fr/usagers/politique-dutilisation/ 


\title{
Émile Zola devant la censure victorienne
}

\author{
Denise Merkle
}

\section{Introduction}

En France, à la fin des années 1880, les Rougon-Macquart ont fait couler beaucoup d'encre. Alain Pagès dans la Bataille littéraire décrit le débat journalistique provoqué par le naturalisme et les romans de Zola, et constate que bien que quelques critiques aient apprécié les qualités poétiques de ses cuvres, d'autres, dont Anatole France fut l'un des plus virulents, dénoncent l'obscénité et l'outrance de la Terre en particulier, avec ses viols, meurtres, incestes, adultères, etc., et son langage grivois. On a pu dire que Nana, qui est l'histoire d'une courtisane, et Pot-Bouille, une dénonciation de l'absence de moralité de la classe bourgeoise parisienne ${ }^{1}$, font preuve d'une ignorance de la réalité bourgeoise de l'époque. Mais ce qui semble surtout déconcerter les critiques littéraires français dans ces romans, c'est la non-application des principes du naturalisme énoncés par Zola. Non seulement les critiques littéraires contestent la valeur artistique et morale des nouveaux romans, mais la censure officielle se donne libre cours.

1. Voir la préface d'Henri Guillemin à Pot-Bouille (Lausanne, Éditions Rencontre, 1961). 
Zola, tout en craignant cette censure, lui est reconnaissant de la publicité gratuite (et des recettes) qu'elle entraîne. Peut-être l'écrivain est-il déjà trop célèbre en France; mais le fait est là: il $n^{\prime} \mathbf{a}$ jamais été poursuivi en justice ${ }^{2}$.

Bien sûr, la censure existe en France. Flaubert n'a-t-il pas été traîné en justice à cause de Madame Bovary? Mais elle ne paralyse pas la création littéraire pour autant. Par contre, en Angleterre, depuis le début du XIX ${ }^{c}$ siècle, la création littéraire est considérablement gênée par une censure omniprésente.

\section{Le «bowdlerisme», la «National Vigilance Association» et les bibliothèques de prêt}

Dans son livre intitulé Garden of Zola, Graham King prévient le lecteur anglophone de la piètre qualité des traductions en anglais (britanniques et autres) des romans de Zola; en fait, il consacre un chapitre entier à l'étude des valeurs victoriennes, à l'influence de ces valeurs sur la création littéraire et sur la traduction. King décrit aussi l'environnement dans lequel Henry Vizetelly veut entreprendre les traductions de Zola; $c^{\prime}$ est une société hautement puritaine, hostile à tout ce qui n'est pas bienséant, où la ligue de moralité (National Vigilance Association), fondée en 1886 afin de promouvoir la pureté sociale, jouit d'un certain pouvoir politique, où les bibliothèques de prêt décident des livres qui seront publiés, et où l'on vit toujours dans l'ombre de Thomas Bowdler.

2. Cependant, dans Pot-Bouille, l'auteur fait allusion à une telle éventualité: «Alors, M. Gourd raconta qu'on était venu de la police, oui, de la police! L'homme du second avait écrit un roman si sale, qu'on allait le mettre à Mazas. - Des horreurs! continua-t-il, d'une voix écœurée. C'est plein de cochonneries sur les gens comme il faut. Même on dit que le propriétaire est dedans; parfaitement, monsieur Duveyrier en personne! Quel toupet!... Ah! ils ont bien raison de se cacher et de ne fréquenter aucun locataire! Nous savons maintenant ce qu'ils fabriquent, avec leurs airs de rester chez eux. Et, vous voyez, ça roule carrosse, ça vend leurs ordures au poids de l'or!» (p. 436) 
Dans la préface à The Family Shakespeare, Bowdler écrit qu'il a supprimé «everything that would raise a blush on the cheeks of modesty" et c'est lui qui, en 1823, a énoncé le principe suivant: «If any word or expression is of such a nature, that the first impression which it excites is an impression of obscenity, that word ought not to be spoken, or written, or printed; and if printed, it ought to be erased» (King, 1978, p. 228). Thomas Bowdler meurt en 1825, mais ses disciples continuent son travail. En 1842, Charles Edward Mudie ouvre la Select Library, qui a le monopole de la production littéraire anglaise entre 1850 et 1890 . Mudie et W.H. Smith sélectionnent tous les manuscrits qui feront partie de leur collection et exigent des éditions de trois volumes et à faible tirage afin de rendre les prix prohibitifs. La majorité des éditeurs coopèrent, car l'arrangement est rentable. Ce système de bibliothèques de prêt devient une forme efficace de censure et réprime la créativité littéraire en Angleterre pendant la deuxième moitié du XIXe siècle.

2. Henry Vizetelly, éditeur des traductions de Zola, et ses démêlés avec la justice britannique

Henry Vizetelly revient dans cette société oppressive après avoir passé une vingtaine d'années à Paris (entre 1860 et 1880), où il était journaliste. C'est à Paris qu'il a fait la connaissance de Zola en 1870 , et, en 1877, qu'il a été témoin du scandale causé par l'Assommoir (roman et pièce) entre autres ouvrages de Zola. Vizetelly s'est vite rendu compte que le succès de cet écrivain était «fondé sur le scandale» (Pagès, 1989, p. 13), et il espérait que ses traductions rapporteraient autant que les œuvres originales.

A son retour à Londres en 1880 , Vizetelly a acheté les droits anglais de l'Assommoir et de Nana. Peu d'éditeurs britanniques osaient toucher aux romans de Zola; ses livres ne se trouvaient donc pas dans les bibliothèques de prêt, seule diffuseur respectable de livres, et les ligues de moralité ne voulaient assurément pas que le peuple ait accès aux éditions bon marché que publiait Vizetelly \& Cie. Par contre, les romans en version originale circulaient librement; seuls les gens instruits issus généralement des classes privilégiées avaient libre accès aux 
romans de Zola. Pour sa part, Vizetelly espérait que les traductions susciteraient l'intérêt et augmenteraient ses recettes. En 1884, il a publié une édition en un seul volume, donc à bon marché, de The "Assommoir» et de Nana. Ces traductions non abrégées étaient donc scandaleuses et elles se vendaient aux kiosques de livres. Quant aux cercles littéraires établis, ils ont honni ces traductions. À cause du grand succès de ces romans en France et de leur grande rentabilité, Vizetelly a acheté les droits d'auteur anglais de toutes les cuvres de Zola; il a pourtant commencé à expurger les romans afin d'échapper à la critique. La version française de Pot-Bouille est publiée en 1882, et c'est en 1885 que sa traduction Piping Hot! est mise sur le marché britannique.

Lors de sa parution en 1887, la Terre a provoqué une critique dans l'ensemble très négative en France. Les "Cinq" dans lcur Manifeste devenu fameux écrivent: «la note ordurière [du romanl était exacerbée encore, descendue à des saletés si basses que, par instants, on se croirait devant un recueil de scatologie ${ }^{3}$ ". Anatole France, quant à lui, écrit dans le Temps que «Jamais homme n'avait fait un pareil effort pour avilir l'humanité, insulter à toutes les images de la beauté et de l'amour, nier tout ce qui est bon et tout ce qui est bien ${ }^{4} \%$. Malgré le scandale français, ou peut-être à cause de celui-ci, Vizetelly fait faire la traduction anglaise de ce roman. Vers la fin 1887, le premier traducteur a refusé de terminer la traduction de la Terre à cause du langage, et un deuxième a réagi de la même façon. C'est pourquoi, Ernest Vizetelly, le fils de Henry, a recommandé la suppression de certains passages et la modification du vocabulaire. En mars 1888, The Soil était prêt pour l'impression. Entre temps, la ligue de moralité avait demandé l'appui du moraliste William Stead, éditeur du Pall Mall Gazette, dans sa campagne de censure des traductions publiées par la maison

3. Voir la note dans laquelle l'auteur cite le Manifeste des Cinq publié dans le Figaro en 1887 (Pagès, La Bataille littéraire, 1989), pp. 16-17.

4. $\quad$ lbid., p. 17. 
Vizetelly. Dans un article de journal, Stead écrit que Vizetelly n'avait aucunement essayé d'expurger les romans de Zola. $\AA$ quoi Vizetelly répond qu'aucune de ses traductions n'est une reproduction fidèle.

Henry Vizetelly a dû comparaître deux fois devant les tribunaux ${ }^{5}$. Le 31 octobre 1888, âgé de 68 ans et en mauvaise santé, il se trouvait à la Old Bailey à Londres, pour défendre sa traduction de Nana, de Pot Bouille et de la Terre. Le procureur, Sir Edward Clarke, a lu quelques passages de The Soil, qui traitaient de l'accouplement d'un bouf et d'une vache, de viol, de meurtre, d'inceste, etc. Au neuvième passage, le jury a refusé d'en entendre davantage $e^{6}$. Vizetelly a fini par plaider coupable. Il a dû payer une amende de 100 livres sterling et a été condamné avec sursis à une peine de douze mois d'emprisonnement. Même la réaction des journaux était vive et témoignait du parti-pris contre Vizetelly? ${ }^{2}$. Ayant besoin d'argent, l'éditeur a continué à vendre les livres qui, d'après lui, n'avaient pas été cités dans le jugement (en fait, le jugement était très vague). Le 30 mai 1889, à la cour criminelle centrale de Londres, a eu lieu un second

5. Pour plus de détails sur les deux procès, voir Graham King, «Scandal!» (1978), pp. 228-254.

6. Geoffrey Robertson explique que la législation de l'Obscene Publications Act (1857) "gave the police powers to take books before local Justices to have them 'forfeited' and destroyed for obscenity. The task of defining obscenity was left to the courts, and chief Justice Cockburn, in the 1868 case of $R$. $v$. Hicklin, obliged with the formula which has influenced the subject ever since: 'I think the test of obscenity is this, whether the tendency of the matter charged as obscenity is to deprave and corrupt those whose minds are open to such immoral influences, and into whose hands a publication of this sort may fall.'" (Robertson, 1989, p. 180).

7. «The Times gloated that 'In future, anyone who publishes translations of Zola's novels and works of similar character will do so at his peril, and must not expect to escape so easily as Mr. Vizetelly.'” (Ibid., p. 181) 
procès. Du fait qu'il avait publié les traductions de The Fortune of the Rougons, The Kill, The Belly of Paris, The Abbé Mouret's Sin, Joy of Life, His Excellency, et Bel Ami (de Maupassant), Vizetelly a plaidé coupable à la recommandation de son avocat. Le procès fut très bref. Comme il n'était pas en mesure de payer une amende, il a été condamné à trois mois de prison pour récidive. Cette fois-ci les journaux se sont tus. Peu après, Vizetelly et Cie a fait faillite.

En 1894, Chatto \& Windus s'est porté acquéreur des droits anglais de Zola et Ernest Vizetelly, qui y travaillait, poursuivit l'expurgation et l'altération de quinze traductions pour ne pas offenser le public (sauf bien entendu la Terre, Nana, et PotBouille qui étaient toujours interdits en Angleterre).

\section{L'autocensure}

Ces trois romans ayant été rejetés par les bibliothèques de prêt, condamnés par la ligue de moralité et interdits par le système judiciaire, l'autocensure prend la relève de la censure. L'autocensure en traduction est un acte conscient ou inconscient de la part du traducteur par lequel il devance la censure officielle. De crainte d'offenser les institutions, le traducteur supprime tout ce qui pourrait leur déplaire; mais, pour ce faire, il doit être imprégné des valeurs de la société dans laquelle il vit. Dans les traductions de Vizetelly, l'autocensure est subtile: des expressions sont atténuées, des phrases modifiées et même des passages assez longs carrément supprimés par le traducteur sans que le lecteur britannique ne s'en rende compte, car ces changements ne sautent pas aux yeux; il aurait fallu comparer l'original avec la traduction pour constater les différences. Vizetelly a lui-même reconnu qu'aucune de ses traductions n'était une reproduction fidèle, et ce sont précisément ces infidélités qui nous intéressent. Nous allons examiner quelques exemples tirés de Pot-Bouille.

Octave Mouret, jeune homme de 22 ans, vient d'arriver à Paris et s'installe dans une maison bourgeoise «comme il faut» où habite une camarade de sa jeunesse à Plassens. Sous peu, il se 
rend compte du comportement louche et hypocrite des locataires, et sans beaucoup résister devient l'un des leurs. Ce roman traite de "l'adultère dans la bourgeoisie ${ }^{8}$ ", de l'hypocrisie sociale (les maîtres peuvent coucher avec les bonnes, mais si par malheur l'une $d^{\prime}$ 'entre elles tombe enceinte ou est malade, elle en est tenue pour responsable et renvoyée) et de "l'insouciance du crime» (Berthe ne se soucie pas du crime que représente l'adultère); $M$. Duveyrier, propriétaire de la maison et magistrat, après avoir renvoyé la piqueuse de bottines (jeune femme non mariée) à cause de son "ventre» énorme trop voyant (et cela quand elle était sur le point d'accoucher), la met en prison pour cinq ans après que celle-ci, sans logement et sans revenu, a tué son nouveau-né). Bref, Zola décrit l'écroulement de la société bourgeoise pourrie et hypocrite dans le Paris des années 1870 et 1880.

Considérons sept catégories d'autocensure, dans ce que le traducteur a décidé d'atténuer, de modifier ou de supprimer dans sa traduction.

\section{a. La représentation de l'acte sexuel}

Amélie et son mari, ouvrier et locataire d'une des chambres sous le toit, veulent passer une nuit ensemble afin de "faire un garçon" (p. 160) comme dit l'époux à deux reprises. M. Vabre, premier propriétaire, et $M$. Gourd, concierge, ne veulent absolument pas que la femme monte dans la chambre de l'ouvrier, car d'abord ils ne croient pas que les deux soient mariés, et ensuite il s'agit d'ouvriers qui, par définition, n'ont pas de morale. En anglais, parler de faire un enfant à l'époque victorienne était trop cru, et le traducteur a atténué en se servant de l'expression euphémistique, "We'll wait till some other time...» (p. 121).

- C'est curieux tout de même... Enfin, puisque monsieur ne veut pas, retourne chez tes maîtres, Amélie. Nous ferons un

8. Henri Guillemin, préface à Pot-Bouille (1961, p. 16). 
garçon une autre fois. Vrai, c'était pour faire un garçon... (p. 160).

It's damned funny all the same. However, as the gentleman objects, you'd better return home, Amelie. We'll wait till some other time... (p. 121).

b. La nudité

«Elle se déshabillait rageusement; puis, se jetant sur le vieux sommier qui craquait, elle tourna le dos. Il dut s'humilier» ( $p$. 162). Ici, la femme (Adèle, une des bonnes de la maison) se déshabille en présence d'un homme (Trublot, invité assidu des familles convenables de la maison), et ce couple non marié partage un lit (il ne s'agit pas d'adultère strictement parlant dans ce cas-ci). Ce passage est supprimé en anglais.

\section{c. La grivoiserie}

L'ouvrier, ivre, parle tout seul dans sa chambre. Il trouve bizarre qu'un homme marié ne puisse pas passer une nuit avec sa femme, tandis que les bourgeois couchent avec les bonnes ou avec leurs maîtresses. En français, il parle de «mettre le nez sous les draps, pour voir» (p. 162), ce qui est évidemment bien trop grossier pour le Victorien; l'anglais rend ses paroles par «poke your nose into all the rooms, and see what you'll see» (p. 122).

\section{d. La grossesse et l'accouchement}

La grossesse et l'accouchement semblent être deux sujets dont les Victoriens ne parlaient pas beaucoup. Être enceinte est rendu par le joli euphémisme «to be in the family way» (p. 174) et l'accouchement par "confinement». Et ils n'entraient pas dans les détails. Par exemple, Octave veut savoir de combien de mois Marie (une de ses maîtresses) est enceinte, car il craint d'être le père. L'Octave de la version anglaise ne s'intéresse aucunement à cette question.

- Je suis enceinte. 
- [...] N'est-ce pas? Marie, si nous savons comment il a pu pousser, celui-là!

- Ça, c'est bien vrai, affirma la jeune femme.

Octave comptait les mois. Elle était enceinte de cinq mois, et de fin décembre à la fin mai, le compte s'y trouvait. Il en fut tout ému; puis, il aima mieux douter; mais son attendrissement persistait, il éprouvait le besoin violent de faire quelque chose de gentil pour les Pichon. (p. 222)

«I am in the family way.»...

«Is it not true, Marie, that we have no idea how it has come about?»

"That is so, indeed,» affirmed the young woman.

It quite affected Octave; and he felt a violent desire to do something nice for the Pichons. (p. 174)

La version française d'un passage que nous ne citerons pas ici à cause de sa longueur décrit en détail l'accouchement d'Adèle, toute seule, ignorante et frissonnante, dans sa chambre glacée (pp. 443-449). Les sept pages que Zola y consacre sont réduites à une et demie dans la version anglaise (pp. 368-369).

\section{e. Les expressions désignant les sacrements}

Les Victoriens censurent les expressions désignant les sacrements. Par exemple, le roman français fait référence à plusieurs reprises au «bon Dieu" pour désigner le sacrement de l'Extrême-onction. L'anglais remplace soit par "[religious] paraphernalia», par «sacrements" ou par des termes qui paraissent plus «convenables» selon le contexte religieux ou autre.

[...] Et l'abbé, après avoir balbutié des prières, s'en alla d'un air de gêne, en remportant le bon Dieu.

- C'est mauvais signe, disait Clémence aux autres domestiques, réunis à la porte de l'antichambre. On ne dérange pas le bon Dieu pour rien... Vous verrez qu'il reviendra dans la maison, avant un an (p. 269).

So, after mumbling a few prayers, Abbé Mauduit withdrew with an air of embarrassment, taking his paraphernalia with him. "It is a bad sign," said Clémence to the other servants, standing in a group at the door of the anteroom. "The 
sacraments are not to be brought for nothing. You will see they will be back in the house before another year goes by.» (p. 215)

\section{f. Le viol}

Dans l'exemple suivant, Berthe se fait violer par Octave. Zola essaie de dépeindre l'aspect agressif de cet acte "Mais il l'empoigna, la jeta sur le lit qu'clle venait d'ouvrir...) (p. 308). Dans un autre exemple, la brutalité de la violence sexuelle, très évidente en français, a été complètement supprimée en anglais:

Alors, il ne parla plus, ayant une revanche à prendre, se disant tout bas, crûment: "Toi, tu vas y passer!" Comme elle refusait de le suivre dans la chambre, il la renversa brutalement au bord de la table; et elle se soumit, il la posséda, entre l'assiette oubliée et le roman, qu'une secousse fit tomber par terre. La porte n'avait pas même été fermée, la solennité de l'escalier montait au milieu du silence. Sur l'oreiller du berceau, Lilitte dormait paisiblement.

Lorsque Marie et Octave se furent relevés, dans le désordre des jupes, ils ne trouvèrent rien à se dire. (p. 115)

Then he left off speaking, having a revenge to take on womankind, and saying coarsely to himself: "You, at any rate, shall succumb!» The door had not even been shut, the solemnity of the staircase seemed to ascend in the midst of the silence. Lilitte was peacefully sleeping on the pillow of her crib. When Marie and Octave rose up, they could find nothing to say to each other. (p. 82)

Zola dépeint non seulement le comportement violent de l'homme, mais il décrit la réaction de la femme face à cette agression:

Elle, silencieuse, le subit sans bonheur. Quand elle se releva, les poignets cassés, la face contractée par une souffrance, tout son mépris de l'homme était remonté dans le regard noir qu'elle lui jeta (p. 308).

Ces phrases, elles aussi, sont supprimées en anglais. Octave, qui est l'agresseur dans trois scènes de viol (deux fois Marie en est la victime), au fond déteste les femmes, comme cette citation le 
révèle: «le dédain féroce qu'il avait de la femme, sous son air d'adoration câline" (p. 308). Pour sa part, la version anglaise se limite à «His moment of triumph had come, but it was no sooner over than all the ferocious disdain of woman, which was hidden beneath his air of wheeling adoration, returned" (p. 250). Bien que la traduction anglaise ait rendu la haine ambiguë d'Octave contre les femmes, le lecteur anglophone perçoit la scène sous un angle différent: il $n^{\prime} y$ a aucune référence à la victime, ni à la violence de l'acte.

\section{g. L'adultère}

Octave veut que Berthe, sa maîtresse, le rencontre dans une chambre d'hôtel pour une heure puisqu'il est impossible d'avoir quelques minutes d'intimité à la maison de la rue de Choiseul. Le traducteur britannique modific le sens en adaptant «to make some assignation out of doors" (p. 262). En général de telles scènes sont supprimées dans la version anglaise, comme ici.

[...] puis, la maison mise de côté, lorsqu'il la conjurait, dehors, de se laisser conduire pour une heure dans une chambre d'hôtel, elle se mettait à pleurer, elle lui disait que, vraiment, il fallait qu'il la respectât bien peu. (p. 323)

[...] then when he implored her to make some assignation out of doors, she would burst into tears, and say that he could really have little respect for her. (p. 262)

Dans l'exemple ci-dessous, en plus de la nudité de la femme et du partage du lit, il est question d'adultère (Berthe, épouse d'Auguste, est la maîtresse d'Octave), ce qui a été supprimé en anglais:

Mal éveillé, frissonnant, il balbutiait à son cou, il l'attirait dans la tiédeur du lit. Elle, déshabillée, avait simplement gardé un jupon et une camisole; et il la sentait comme nue, ses cheveux déjà noués pour la nuit, ses épaules encore tièdes du peignoir dont elle sortait.

- Bien vrai, je te renverrai au bout d'une heure... Reste! (pp. 312-313) 
On sait qu'à cette époque l'adultère de la part de l'époux est sans conséquence, alors que de la part de la femme il peut mener au divorce. L'aristocratic le pratique, mais en cachette (Pearsall, 1971, pp. 181-186).

\section{Conclusion}

Il serait possible de relever d'autres exemples aussi riches en contenu socioculturel. Ceux que nous venons de présenter donnent une idée de la pratique courante dans la traduction victorienne des œuvres de Zola. Nous constatons que, lorsqu'il s'agit de viol, d'adultère ou de nudité, le traducteur a tendance à supprimer les passages incriminants. Lorsqu'il s'agit de grossesse, d'accouchement, d'expressions sacrilèges et de grivoiscrie, le traducteur a tendance à modifier le texte ou à se servir d'euphémismes. Ce que l'autocensure a épargné est parfois intéressant: par exemple, une tentative de suicide est décrite en détail sans que la traduction ne soit modifiée. Le vocabulaire et les scènes qui ont été modifiés, expurgés ou retenus révèlent les valeurs du public visé ainsi que celles du traducteur et de son éditeur. Nous l'avons vu, la traduction de Piping Hot! est particulièrement révélatrice des valeurs puritaines de l'époque. Il est cependant curieux que, ces trois œuvres ayant été expurgées, la ligue de moralité n'ait pas été satisfaite et ait voulu les interdire. Il semble que ce qui a tant déconcerté les Victoriens allait bien au-delà du langage. Sans doute Zola troublait-il trop les sensibilités victoriennes par la représentation de certains maux sociaux tels que l'infanticide, la pauvreté, la prostitution, la spéculation, les maladies vénériennes et l'adultère, ces maux dont précisément la société victorienne souffrait et que les institutions refusaient de voir.

Université Laurentienne

Mes remerciements à $\mathrm{M}^{\text {me }}$ Françoise Arbuckle pour son soutien. 


\section{Références}

COLBURN, William E. (1968). "Victorian Translations of Zola", Studies in the Literary Imagination, (George State College) I (2), pp. 23-32.

CURTIS, Simon (1983). «Vizetelly \& Co», PN Review (32), pp. 2831.

GRIEST, Gunivere (1970). Mudie's Circulating Library. Indiana, Indiana U. Press.

KING, Graham (1978). Garden of Zola (Émile Zola and his Novels for English Readers). New York, Barnes \& Noble.

LAROSE, Robert (1987). Théories contemporaines de la traduction. Québec, Presses de l'Université du Québec.

MITTERAND, H. et B. H. Bakker, dir. (1978). Correspondance VI - 1887-1890. Montréal, Presses de l'Université de Montréal.

PAGÈS, Alain (1989). La bataille littéraire (Essai sur la réception du naturalisme à l'époque de Germinal). Paris, Librairie Séguier.

PEARSALL, Ronald (1971). The Worm in the Bud: The World of Victorian Sexuality. London, Penguin Books.

(1976). Public Purity, Private Shame (Victorian Sexual Hypocrisy Exposed). London, Weidenfeld \& Nicolson.

PERRIN, Noel (1993). Dr. Bowdler's Legacy: A History of Expurgated Books in England \& America. London, Macmillan \& Co., 1970; London, Godine 1969, rev. 1993.

ROBERTSON, Geoffrey, Q.C., (1989). Freedom, the Individual and the Law. London, Penguin. 
VIZETELLY, Ernest A. (1904). Émile Zola, Novelist and Reformer. London, John Lane, The Bodley Head.

VIZETELLY, Henry (1893). Glances Back through 70 Years. London, Kegan, Paul, Trench, Trubner \& Co.

ZOLA, Émile (1963). La Terre. Lausanne, Éditions Rencontre.

(1960). Nana. Lausanne, Éditions Rencontre.

(1885). Piping Hot! Translation of Pot-Bouille. London, Vizetelly \& Co.

(1961). Pot-Bouille. Lausanne, Éditions Rencontre.

RÉSUMÉ: Émile Zola devant la censure victorienne - Cette étude donne un aperçu sommaire de quatre formes prises par la censure victorienne - la ligue de moralité ( National Vigilance Association»), les bibliothèques de prêt («circulating libraries»), la justice et l'autocensure («bowdlerism») - et leurs effets sur la traduction des romans de Zola. Les œuvres de ce dernier ont connu un succès de scandale et ont provoqué un débat journalistique en France, mais Zola n'a jamais été traîné en justice à cause de ses écrits. Par contre, en Angleterre, les cercles littéraires établis honnissent les traductions victoriennes de ses œuvres et la ligne de moralité appuyée par le moraliste Stead lance une campagne de censure des traductions de Zola. L'éditeur de ces traductions, Henry Vizetelly, a dû comparaître deux fois devant les tribunaux et les deux fois il a été condamné. Non seulement il dut payer une amende et passer trois mois en prison, mais encore Nana, Piping Hot! (Pot-Bouille) et The Soil (la Terre) ont été interdits, même si ces trois traductions avaient - comme les autres - été «autocensurées» par les traducteurs et l'éditeur avant la parution. Il est dès lors évident que ce sont les idées réformatrices de Zola et le lectorat visé (petite bourgeoisie et classes populaires) qui ont motivé l'opposition des pouvoirs constitués de l'Angleterre de l'époque aux romans de Zola.

ABSTRACT: Émile Zola and Victorian Censorship - This paper is an introduction to four aspects of Victorian censorship - the National Vigilance Association, circulating libraries, the courts, and bowdlerism - and their impact on the translation of Zola's novels. In France, scandal ensured success, and the literary value of Zola's works was 
hotly debated by the press, but Zola was never taken to courts over his writings. On the contrary, in England, established literary circles held the Victorian translations of his novels in contempt and the National Vigilance Association, supported by the moralist Stead, launched a campaign to censor the translations. Henry Vizetelly, the editor of these translations, was required to appear two times before the courts to defend them, and both times he lost his case. Not only did he have to pay a fine and spend three months in prison, but Nana, Piping Hot! and The Soil were banned, even though these three translations had been bowdlerized by the translators and the editor prior to their initial publication. It becomes clear that Zola's interest in social reform and the intended readership - lower and middle classes - motivated the official opposition to these translations in England. 